\title{
A Correlated APT and TEM Approach to Understand Nanostructured Ferritic Alloys
}

\author{
M. K. Miller, C. M. Parish, and L. Yao
}

Materials Science and Technology Division, Oak Ridge National Laboratory, Oak Ridge, TN 37831

Nanostructured ferritic alloys (NFA) are a subcategory of oxide-dispersion strengthened (ODS) steels being evaluated for applications in future advanced energy systems under extreme conditions. These complex ultrafine-grained materials exhibit excellent resistance to high temperature creep and are tolerant to high doses of irradiation. In order to understand these unique properties, a correlated approach has been used to characterize the microstructure over multiple length scales, primarily with a combination of atom probe tomography (APT) and scanning transmission electron microscopy (STEM), but also with several other techniques, including neutron scattering and positron annihilation spectroscopy. Each of these techniques has a range of applicability and inherent limitations, especially for complex commercial materials, such as this NFA. There are many advantages of applying multiple techniques to quantify the microstructure of complex materials. For example, the morphology and composition of ultrafine-scale features estimated from APT data can be used to produce an accurate model of the microstructure that can be used to correctly interpret the scattering data. Phase identification is performed from diffraction information in the (S)TEM and from the composition estimates in APT. Although the constantly changing specimen thickness is not ideal for (S)TEM, needleshaped APT specimens and $360^{\circ}$ rotation TEM holders can be used for combined electron tomography and APT on the same region of the specimen.

The solute distribution in an ultrafine-grained 14YWT NFA specimen that was Pt ion irradiated to a dose of $25 \mathrm{dpa}$ at $750^{\circ} \mathrm{C}$ is shown in a series of energy-filtered TEM (EFTEM) images in Fig. 1. The FeM EFTEM map reveals two populations of precipitates on the grain boundary (GB). A series of $\sim 5-10$ $\mathrm{nm}$ diameter precipitates and a coarser $\sim 20 \mathrm{~nm}$ diameter precipitate enriched in $\mathrm{Ti}$ and $\mathrm{N}$. Previous characterizations have identified these precipitates as $\mathrm{Y}_{2} \mathrm{Ti}_{2} \mathrm{O}_{7}$ with a cubic $\left(\mathrm{Fd}_{3} \mathrm{~m}\right)$ structure (i.e., pyrochlore) and $\mathrm{Ti}(\mathrm{N}, \mathrm{C}, \mathrm{O})$ with the $\mathrm{NaCl}$ structure, respectively.[1] Segregations of $\mathrm{Cr}$ and $\mathrm{Ti}$ to the GBs are evident in the $\mathrm{Cr}-\mathrm{L}$ and Ti-L maps. These precipitates and solute segregation effectively pin the GB, which along with the slow diffusion in this NFA material, are responsible for the excellent creep properties observed at high temperatures.

This mechanically alloyed NFA also contains a high number density of Ti-Y-O-enriched nanoclusters both in the grain interior and on the GBs. These precipitates have been termed nanoclusters to distinguish them from the other populations of precipitates. The nanoclusters are extremely stable against high temperature annealing treatments and high doses of neutron and ion irradiation, as shown for and 14YWT NFA that was Pt ion irradiated to a dose of $215 \mathrm{dpa}$ at $750{ }^{\circ} \mathrm{C}$ in Fig. 2 . As the $1-2 \mathrm{~nm}$ diameter nanoclusters are substantially smaller than the thickness of a typical TEM foil, the observed contrast is affected by the nanoclusters, the ferro-magnetic matrix, the position of the nanocluster within the thickness of the foil, the surface condition of the foil (including oxidation), hydrocarbon contamination, and surface vacancy content, the imaging conditions used, and the viewing direction. The difficulty of defining the precise nanocluster- matrix interface due to its diffuse nature at the atomic scale, and the possibility of small ion trajectory aberrations due to the different evaporation fields of the matrix and the nanoclusters, also complicate APT composition estimates. Therefore, the non-exclusive 
possibilities of the precise nature of the nanoclusters include non-stoichiometry, high vacancy content, disordered or possibly amorphous precipitates, metastable embryos, or even a new alloying state.[2]

References:

[1] MC Brandes, L Kovarik, MK Miller, MJ Mills, J. Mater. Sci. 47 (2012) 3913-3923.

[2] Research supported by the U.S. Department of Energy (DOE), Basic Energy Sciences (BES), Materials Sciences and Engineering Division, and through a user project supported by ORNL's Shared Research Equipment (ShaRE) User Program, which is also sponsored by DOE-BES.

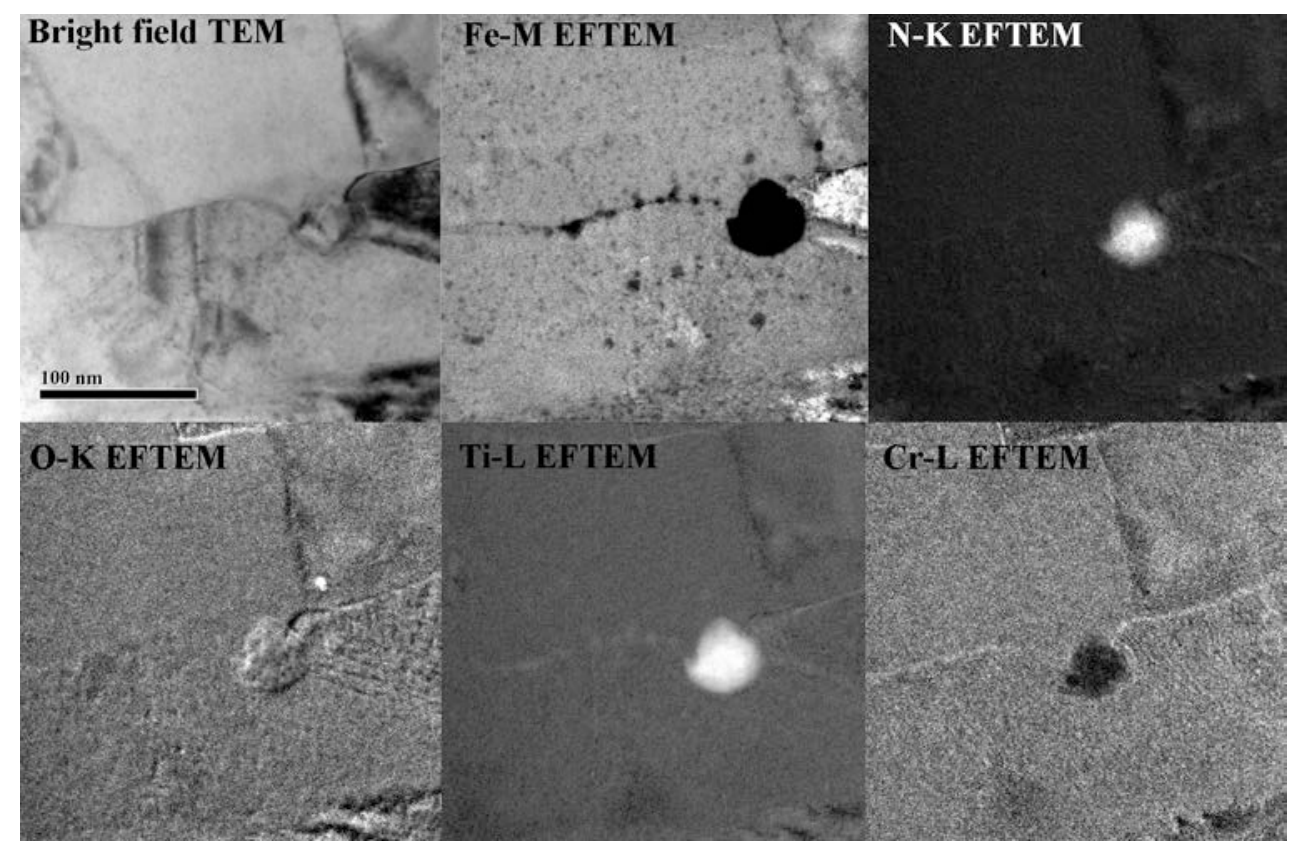

Figure 1. EFTEM maps of a 14YWT NFA Pt ion irradiated to a dose of 25 dpa at $750{ }^{\circ} \mathrm{C}$.

Figure 2. APT atom map and $0.8 \% \mathrm{TiO}$ isoconcentration surfaces showing the distribution of 1-2 nm diameter nanoclusters in the grain interior (grey) and on GBs (blue) in 14YWT NFA Pt ion irradiated to a dose of 300 dpa at $750{ }^{\circ} \mathrm{C}$.

1

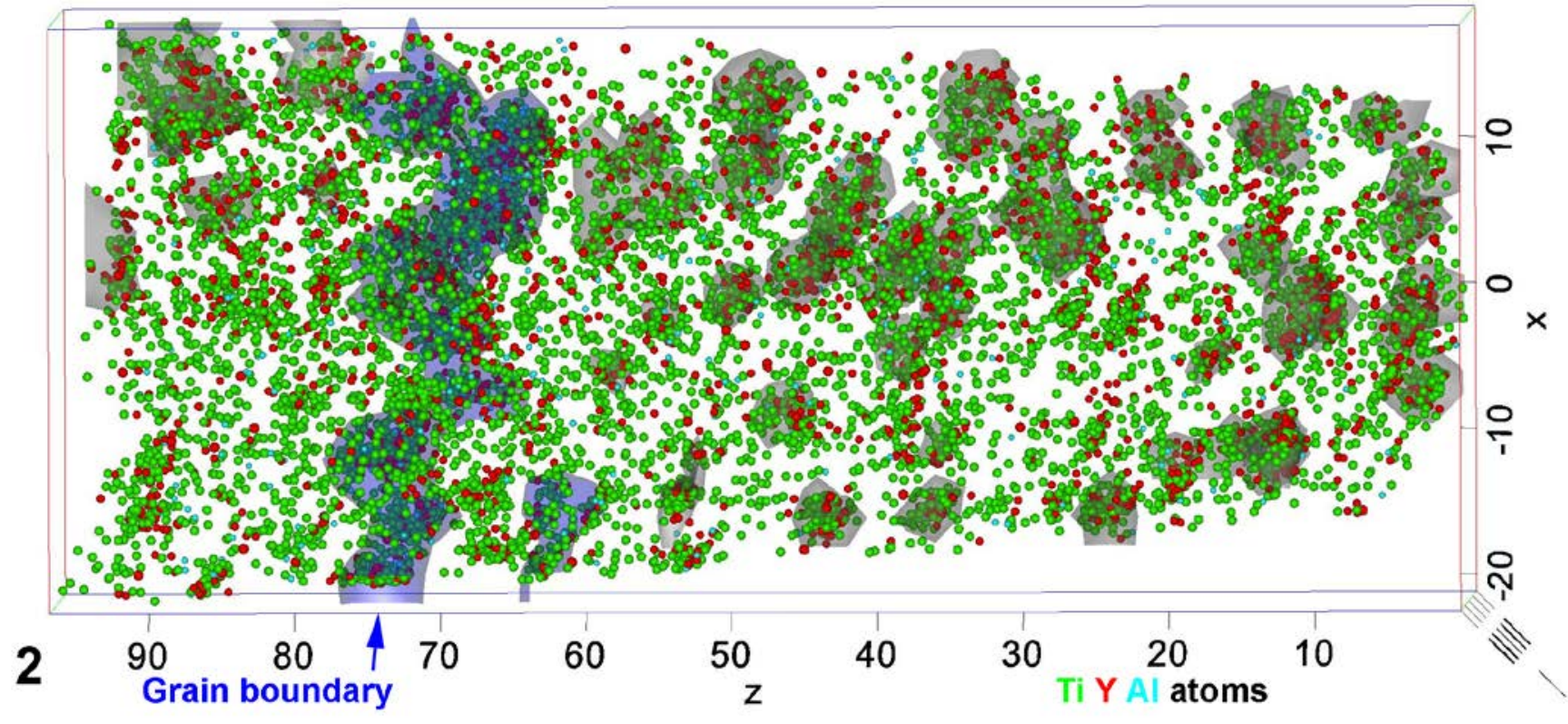

